\title{
ANÁLISIS PALINOFACIAL DE LA ALOFORMACIÓN PLAYA DEL ZORRO (MIOCENO TARDÍO/PLIOCENO), VALLE DEL CAJÓN, CATAMARCA, ARGENTINA
}

\author{
MARICEL YANINA HORN \\ Centro de Ecología Aplicada del Litoral, Universidad Nacional del Nordeste, CONICET, Ruta Provincial N5, \\ Corrientes (3.400), Argentina.yaninahorn@yahoo.com \\ MARCELO ADRIÁN MARTÍNEZ \\ Departamento de Geología, Instituto Geológico del Sur, Universidad Nacional del Sur, CONICET, San Juan 670, \\ B8000ICN Bahía Blanca, Argentina.martinez@criba.edu.ar \\ LUISA MATILDE ANZÓTEGUI \\ Centro de Ecología Aplicada del Litoral, Universidad Nacional del Nordeste, CONICET, Ruta Provincial ํ5, \\ Corrientes (3.400), Argentina. luisaanzotegui@yahoo.com.ar
}

\begin{abstract}
PALYNOFACIES ANALYSIS OF PLAYA DEL ZORRO ALLOFORMATION (LATE MIOCENE/PLIOCENE) CAJON VALLEY, CATAMARCA, ARGENTINA. This paper presents the palynofacies analysis results from La Cascadita locality of Playa del Zorro Alloformation (late Miocene-Pliocene), being the first palynological study of the Neogene outcrops of the Cajon Valley, northwestern Argentina. Eleven palynological and organic matter samples were analyzed, only three of them containing palynomorphs (spores of Glomus sp. and Lycoperdon sp., Chlorophyta algae colonies, spores Zygnemataceae and few angiosperm pollen grains). Four types of organic matter were identified: phytoclasts (translucent and opaque), palynomorphs, amorphous organic matter and zooclasts. Given the overall frequency of these types and relative frequencies of the particles that constitute them, two palynofacies can be distinguished. The Palynofacies Type I is characterized by the relatively high frequency semi-opaque and equidimensional opaque phytoclasts, reduced organic amorphous matter and low representativeness of palynomorphs; suggesting oxidizing environments, poor transport and high-energy environment. The Palynofacies Type II is characterized by high relative frequency of palynomorphs and amorphous organic, and have the lower values of phytoclasts, which suggests an environment with more reducing conditions than those of the Palynofacies I, temporarily anoxic and shallow. The climate regime would have been warm, semi-arid with a marked dry seasonality.
\end{abstract}

Key words: palynofacies, paleoenvironmental inferences, Neogene, northwestern Argentina.

RESUMO - No presente trabalho são apresentados os resultados de análises de palinofácies realizadas na localidade La Cascadita da Aloformação Playa del Zorro (Mioceno tardío-Plioceno), sendo este o primeiro estudo palinológico dos afloramentos neógenos do Valle del Cajón, noroeste da Argentina. Foram analisadas a matéria orgânica e palinológica de onze amostras sedimentares, entre as quais apenas três encontraram palinomorfos (esporos de fungos de Glomus sp. e Lycoperdon sp., colônias de algas Chlorophyta, esporos de Zygnemataceae e escassos grãos de pólen de angiospermas). Foram identificados quatro tipos de matéria orgânica: fitoclastos (translúcidos e opacos), palinomorfos, matéria orgânica amorfa e zooclastos. Tendo em conta as frequências gerais e relativas dos componentes orgânicos particulados, foi possível distinguir duas palinofácies ao longo dos níveis. A Palinofácies Tipo I, que se caracteriza pela alta frequência relativa de fitoclastos semiopacos e opacos equidimensionais, matéria orgânica amorfa reduzida e baixa representatividade de palinomorfos, sugere ambientes oxidantes, escasso transporte e alta energia do meio. A Palinofácies Tipo II se caracteriza pela alta frequência relativa de palinomorfos e matéria orgânica amorfa bem preservada, e por ter os mais baixos valores de fitoclastos, permitindo supor um ambiente com condições de depósito mais redutoras que as da Palinofacies I, temporariamente anóxico e de pouca profundidade. O regime climático teria sido quente, semiárido com uma marcada sazonalidade seca.

Palavras-chave: palinofácies, inferências paleoambientais, Neógeno, noroeste da Argentina.

\section{INTRODUCCIÓN}

El Valle del Cajón, es una depresión tectónica de sentido Norte-Sur comprendido en la provincia geológica de Sierras Pampeanas Occidentales. Geográficamente se encuentra ubicado en la zona noroeste de la Provincia de Catamarca (Argentina) y se sitúa entre la Sierra del Cajón o de Quilmes al este y la Sierra de Chango Real al oeste (Vides \& Bazán, 2004) (Figura 1). En el valle propiamente dicho, afloran sedimentitas del Neógeno, donde se reconocen: arenisca, 

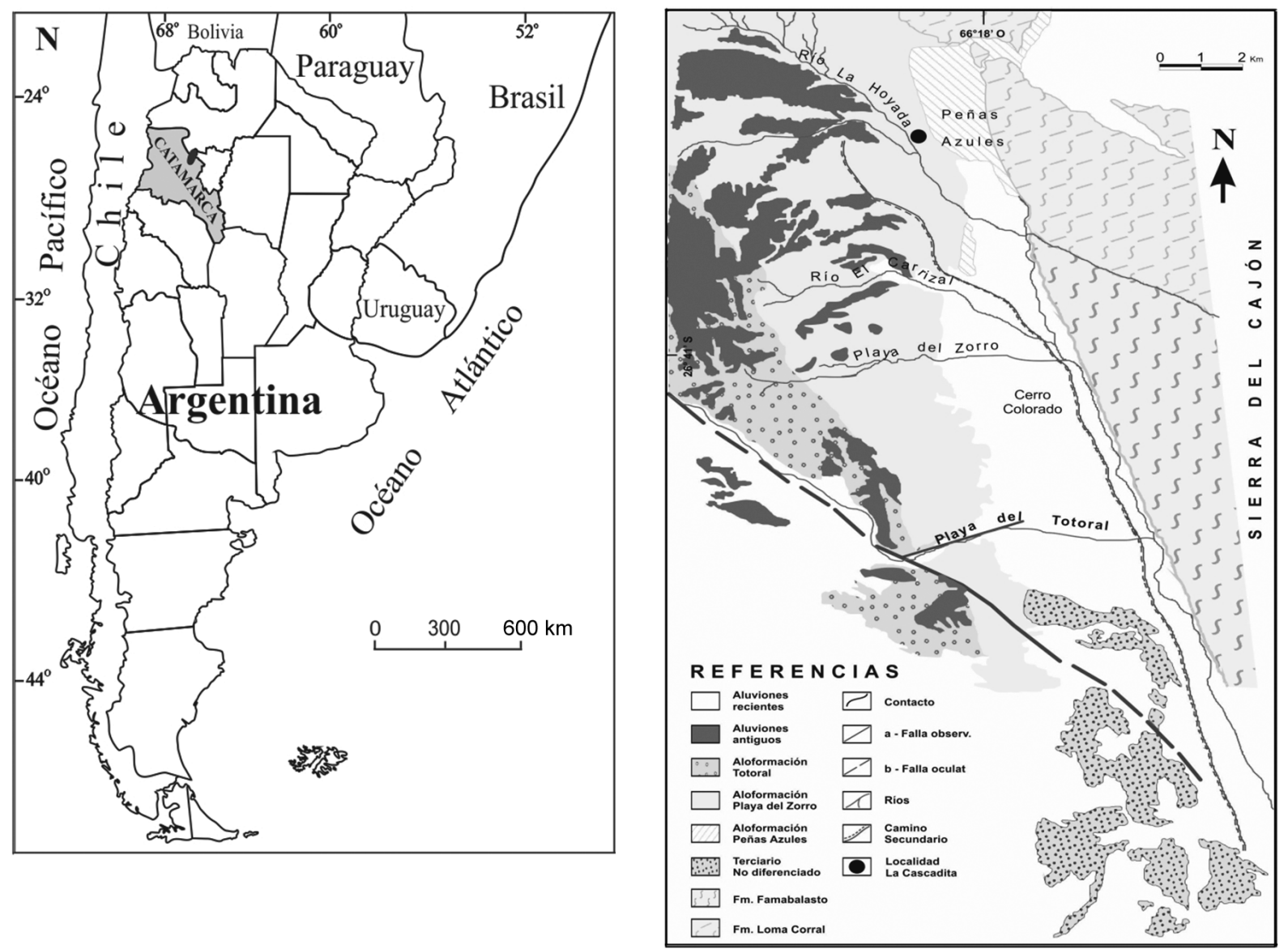

Figura 1. Mapa geológico del valle del Cajón y ubicación del área de estudio (Morton, 2004; modificado de Bossi et al., 1992).

Figure 1. Geological map Cajon valley and location of the study area (taken from Morton, 2004, modified Bossi et al., 1992).

conglomerado, limolita y arcilita entre las que se intercalan numerosas capas de toba y escasa roca volcánica (Vides \& Bazán, 2004). Turner (1973), define a esta sucesión como Formación El Morterito, integrante del grupo El Bolsón. Posteriormente, Bossi et al. (1992) dividen a los afloramientos neógenos de este valle, en tres unidades discordantes entre sí: Aloformación Peñas Azules (Secuencia I), Aloformación Playa del Zorro (Secuencia II) y Aloformación El Totoral (Secuencia III), todas ellas de origen continental. De acuerdo a las características sedimentológicas (textura, estructuras mecánicas y químicas) Bossi et al. (1992, 2000); Vides (2009) y Vides \& Bazán (2004), identificaron distintos subambientes: lago (limolita laminada o estratificada fina, con bivalvos, gastrópodos, ostrácodos, escamas de peces, con escasa participación de arena ondulítica), orla de lago (limolita y arenisca fina con cuerpos elongados de arenisca gruesa, sedimentos costeros bioturbados a veces con grandes concentraciones de pelecípodos y gastrópodos), canales fluviales de alta energía (arenisca gruesa y conglomerado) y canales fluviales de baja energía (con arenisca fina), interfluvios activos (arenisca fina, con laminación ondulada y bioturbación vegetal de pedotúbulos y rizoconcreciones subordinadas) e interfluvios pasivos (arenisca fina macizas con estratificación gruesa, rizoconcreciones, calcretes y en casos extremos concreciones de yeso).

En el presente trabajo se dan a conocer los resultados del análisis palinofacial realizado en la localidad La Cascadita de la Aloformación Playa del Zorro (Secuencia II) (Bossi et al., 1992), de edad estimada Mioceno tardío/ Plioceno (entre 7-3Ma) (Georgieff et al., 2012), siendo este el primer estudio palinológico de los afloramientos neógenos del Valle del Cajón (Figura 1). El registro fósil de dicha aloformación, está representado por invertebrados (Morton, 2004; Morton \& Herbst, 2012), vertebrados (Bossi et al., 1992; Esteban, 1999; Esteban \& Nasif, 1996, entre otros), y en menor medida por vegetales (Anzótegui et al., 2007). El registro palinológico de otras unidades litológicas regionales coetáneas, proceden de la Formación Chiquimil (Mioceno tardío) (Mautino, 2007, 2010, 2011, Mautino \& Anzótegui, 1998, 2000, 2002a,b, 2014), y de la Formación Palo Pintado (Mioceno tardío/Plioceno) (Anzótegui \& Cuadrado, 1996; Acevedo et al., 1997, 1998; Mautino, 2007; Horn et al., 2011a,b; Horn, 2014), cuyos sedimentos afloran en el Valle de Santa María, Provincia de Catamarca y en el sector sur del Valle Calchaquí, Provincia de Salta, respectivamente. 


\section{Área de estudio}

La Aloformación Playa del Zorro (Secuencia II), es la que presenta las mejores exposiciones que ocupan la parte central del valle. La sección tipo se encuentra a la altura del Puesto Molle Grande con un espesor de $1.288 \mathrm{~m}$, incluyendo sus contactos disconformes superior e inferior, y se divide a su vez, en cuatro subsecuencias (Bossi et al., 1992).

Bossi et al. (2000) a partir del análisis de paleocorrientes y litoestratigráfico, concluyen que la sucesión neógena del Valle del Cajón permite inferir condiciones cambiantes en el sistema de dispersión aluvial, controladas por el continuo ascenso de la Sierra de Quilmes que separó los valles del Cajón (al oeste) y de Santa María (al este). Así la Aloformación Playa del Zorro contiene las facies fluviales de mayor sinuosidad, etapas de inundación, formación de lagos y escaso aporte sedimentario. La localidad en estudio, está situada entre las localidades Ovejería Chica y Peñas Azules, sobre el margen de un afluente del río La Hoyada (Figura 1). El perfil realizado en esta localidad consta de $60 \mathrm{~m}$ de espesor donde predominan potentes niveles de arenisca fina a mediana, intercalados con niveles de pelita $(3 \mathrm{~m})$ y arena limosa de poco espesor (1,5-5 $\mathrm{m})$. En la base y en la sección media del perfil se encuentran niveles de arena tobácea de 3-4 m de espesor (Figura 2).

\section{MATERIAL Y MÉTODOS}

Se analizaron 11 muestras sedimentarias (pelita, arena limosa y arena tobácea), que fueron procesadas para el análisis palinológico en el laboratorio del Centro de Ecología Aplicada del Litoral y para el análisis de palinofacies en el Departamento de Geología de la Universidad de Buenos Aires. En ambos casos las muestras fueron tratadas con ácido clorhídrico y ácido fluorhídrico; para el análisis de la materia palinológica (palinofacies), el residuo fue filtrado en malla de $10 \mu \mathrm{m}$. Los preparados forman parte de la Colección Paleontológica de la Universidad Nacional del Nordeste "Dr. Rafael Herbst", bajo el acrónimo CTES-PMP y con los números 3074-3078, 3080-3082 y 3084-3087. Los mismos fueron estudiados con microscopio óptico de luz transmitida, modelo Nikon Eclipse-100 con cámara digital incorporada.

Se contabilizaron entre 300 y 500 partículas por cada muestra palinológica. Siguiendo a Tyson (1995), se clasificaron en cuatro tipos principales de materia orgánica palinológica (Tabla 1). De la totalidad de las muestras (M) procesadas, solo en 2 de ellas (M 7 y 8) se logró contabilizar 100 palinomorfos representados por esporas de hongos, colonias de algas Chlorophyta, esporas de Zygnemataceae y escasos granos de polen de angiospermas.

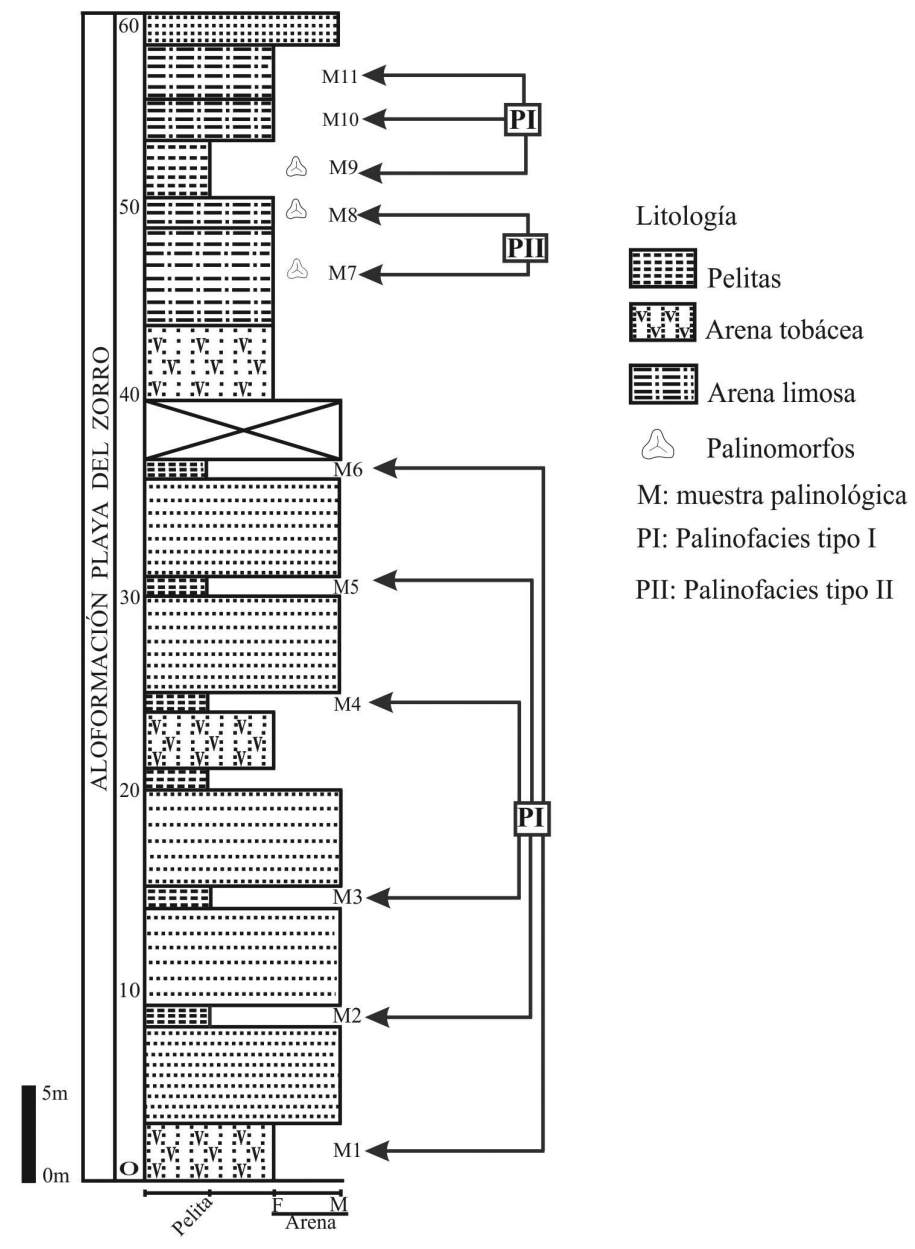

Figura 2. Columna estratigráfica de la localidad la Cascadita. Se señalan la ubicación de las muestras estudiadas y las palinofacies identificadas.

Figure 2. Stratigraphic column of the locality La Cascadita, showing the location of studied samples and identified palynofacies. 
Tabla 1. Clasificación de los tipos de materia orgánica identificados.

Table 1. Classification of the organic matter types identified.

\begin{tabular}{ll}
\hline & Fitoclastos translúcidos \\
& Bioestructuados: hifas de hongos, traqueidas, cutículas, tejidos y otros \\
& No bioestructurados: degradados y semiopacos traslúcidos en los bordes \\
FITOCLASTOS & Fitoclastos opacos \\
& Equidimensionales: redondeados-subredondeados y angulares \\
& En tablilla: bioestructurados y no bioestructurados \\
Charcoal \\
MATERIA ORGÁNICA AMORFA & Granular, granular-esponjosa, esponjosa, membranosa, fibrosa y otros \\
ZOOCLASTOS & Esporomorfos \\
\hline
\end{tabular}

\section{RESULTADOS}

Se logró identificar cuatro tipos de materia orgánica palinológica: fitoclastos, palinomorfos, materia orgánica amorfa y zooclastos (Tabla 1). Las frecuencias relativas generales que cada uno de estos tipos presentan en las muestras estudiadas se exponen en la Tabla 2 y Figura 3. Considerando las frecuencias generales y las frecuencias relativas de las partículas que conforman los tipos de materia orgánica mencionados, es posible agrupar los niveles sedimentarios en dos tipos de palinofacies (Tabla 3). La palinofacies Tipo I se reconoce en las muestras $\mathrm{N}^{\circ} 1,2,3,4,5,6,9,10$ y 11 , mientras que la Palinofacie Tipo II se identifica en las muestras $\mathrm{N}^{\circ} 7$ y 8 (Figura 1).

\section{Palinofacies tipo I (M 1-6 y 9-11)}

Se caracteriza por la alta frecuencia relativa de fitoclastos (76,6\% a 97,66\%). Estos comprenden fitoclastos traslúcidos $(21,84 \%$ a $58,77 \%)$, predominando (en general ampliamente) los no-bioestructurados sobre los bioestructurados. Entre los fitoclastos opacos $(41,23 \%$ a $78,16 \%)$ (Figuras 4D-F), los equidimensionales angulares se presentan con mayor frecuencia (hasta 63,97\%) (ver Tabla 3). La materia orgánica amorfa constituye menos del $20 \%$ del contenido orgánico total, representada principalmente por agregados granulares, esponjosos y membranosos. Los palinomorfos se encuentran con baja representatividad (hasta 5,4\%). Los zooclastos también son escasos (hasta $0,4 \%$ ) y están presentes en las muestras 1-5 y 9. Litofacies asociadas: arena tobácea, pelitas, $\mathrm{y}$ arena limosa.

\section{Palinofacies tipo II (M 7 y 8)}

Se caracteriza por las altas frecuencias relativas de palinomorfos (22\% y $54 \%$ respectivamente) y materia orgánica amorfa ( $31,4 \%$ y $16 \%$ respectivamente), y a su vez presentan los más bajos valores de fitoclastos (46,4\% y $30 \%)$ registrados. Los palinomorfos en la M 7 están representados por esporas de hongos asignadas a Glomus sp. (Glomaceae) $(8,42 \%)$ (Figura $4 \mathrm{H})$ y Lycoperdon sp. (Lycoperdaceae) (81,05\%) (Figura 4I), esporas de Zygnemataceae (10\%) identificadas como Ovoidites sprigii (Cookson \& Dettmann) Zippi, 1998 (Figura 4O) y Ovoidites parvus (Cookson \& Dettman) Nakoman, 1966 (Figura 4N) y granos de polen de Equisetosporites sp. (Ephedraceae) (1,05\%) y Chenopodipollis chenopodiaceioides (Martin) Truswell et al., 1985 (Chenopodiaceae) (6,32\%). En tanto que, en la M 8 se registra Botryococcus braunii Kützing, 1849, en muy alta frecuencia relativa $(94,44 \%$ ) (Figuras $4 \mathrm{~J}-\mathrm{M})$.

Tabla 2. Porcentajes generales de los tipos de materia orgánica identificados en cada una de las muestras estudiadas.

Table 2. Percentage of general organic matter types identified in each of the samples studied.

\begin{tabular}{|c|c|c|c|c|c|c|}
\hline 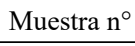 & Litofacies asociada & Fitoclastos \% & Amorfo \% & Palinomorfos \% & Zooclastos \% & Total partículas \\
\hline M 11 & Arena limosa & $85 \%$ & $9,4 \%$ & $5,6 \%$ & 0 & 500 \\
\hline M 10 & Arena limosa & $84,4 \%$ & $14,8 \%$ & $0,8 \%$ & 0 & 500 \\
\hline M 9 & Pelita & $76,6 \%$ & $18,4 \%$ & $4,8 \%$ & $0,2 \%$ & 500 \\
\hline M 8 & Arena limosa & $30 \%$ & $16 \%$ & $54 \%$ & 0 & 500 \\
\hline M 7 & Arena limosa & $46,44 \%$ & $31,4 \%$ & $22 \%$ & $0,2 \%$ & 500 \\
\hline M 6 & Pelita & $97,66 \%$ & $1,67 \%$ & $0,67 \%$ & 0 & 300 \\
\hline M 5 & Pelita & $92,4 \%$ & $6,2 \%$ & $1,2 \%$ & $0,2 \%$ & 500 \\
\hline M 4 & Pelita & $94,4 \%$ & $4,8 \%$ & $0,6 \%$ & $0,2 \%$ & 500 \\
\hline M 3 & Pelita & $92,2 \%$ & $5,4 \%$ & $2 \%$ & $0,4 \%$ & 500 \\
\hline M 2 & Pelita & $89,8 \%$ & $9,4 \%$ & $0,6 \%$ & $0,2 \%$ & 500 \\
\hline M 1 & Arena tobácea & $77,6 \%$ & $18,8 \%$ & $3,4 \%$ & $0,2 \%$ & 500 \\
\hline
\end{tabular}




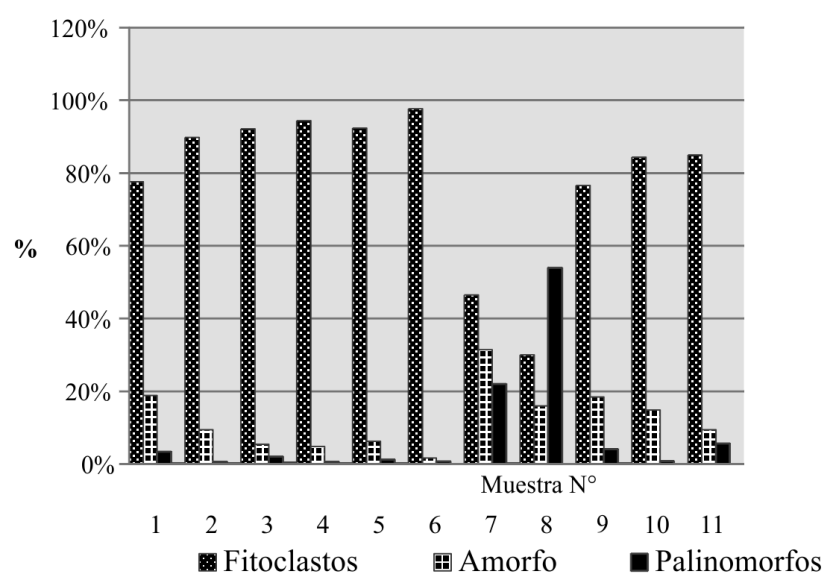

Figura 3. Gráfico de columnas donde se representan los porcentajes generales de los tipos de materia orgánica identificados en las muestras analizadas (Tabla 2).

Figure 3. Column chart showing the general percentages of organic matter types identified in the samples analyzed (Table 2).
Los fitoclastos traslúcidos bioestructurados están representados por hifas, traqueidas, cutículas y otros (Figuras 4A-C). En la M 8 las cutículas presentan una alta frecuencia relativa $(72,09 \%)$ en relación a su frecuencia en la M 7 (5,50\%). En cambio los fitoclastos traslúcidos no bioestruturados semiopacos, (aquellos opacos en el centro y traslúcidos en los bordes) están mejor representados en la M 7 (57,80\%). Entre los fitoclastos opacos, los equidimensionales angulares poseen la mayor frecuencia relativa en ambas muestras, siendo más elevada en la $\mathrm{M}$ $7(81,30 \%)$. La materia orgánica amorfa está representada por partículas granulares, granular-esponjosas, esponjosas, membranosas (Figura 4G), fibrosas y otras. En la M 7 toman mayor representatividad las partículas fibrosas $(38,85 \%)$ y membranosas $(34,39 \%)$ en tanto en la M 8 las partículas membranosas y esponjosas presentan la misma frecuencia relativa $(36,25 \%)$ y son las más numerosas en relación a los restantes tipos. Los zooclastos se presentan sólo en la M 7. Litofacies asociada: arena limosa.

Tabla 3. Resultados del análisis palinofacial. Porcentajes generales de los tipos de materia orgánica identificados y representatividad de los diferentes grupos de partículas. Símbolos: $\bullet=0-10 \% ; \quad=10,5-50 \% ; \boldsymbol{\Delta}=50,25-100 \%$.

Table 3. Results of palynofacial analysis. Overall percentages of the types of organic matter identified and representation of different groups of particles. Symbols: $\bullet=0-10 \% ; \quad=10.5-50 \% ; \boldsymbol{\Delta}=50.25-100 \%$.

\begin{tabular}{|c|c|c|c|c|c|c|c|c|c|c|c|}
\hline \multirow[b]{2}{*}{ TIPOS DE MATERIA ORGÁNICA \% } & \multicolumn{11}{|c|}{ MUESTRAS-PALINOFACIES } \\
\hline & 1-P1 & 2-P1 & 3-P1 & 4-P1 & $5-\mathrm{P} 1$ & 6-P1 & 7-P2 & $8-\mathrm{P} 2$ & 9-P1 & $10-\mathrm{P} 1$ & 11-P1 \\
\hline FITOCLASTOS & 77,6 & 89,8 & 92,2 & 94,4 & 92,4 & 97,66 & 46,4 & 30 & 76,6 & 84,4 & 85 \\
\hline Fitoclastos traslúcidos & 49,74 & 33,85 & 44,03 & 40,89 & 28,35 & 21,84 & 46,98 & 86 & 51,17 & 58,77 & 33,88 \\
\hline \multicolumn{12}{|l|}{ Bioestructurados } \\
\hline Cutículas & - & - & $\bullet$ & - & - & - & $\bullet$ & $\boldsymbol{\Delta}$ & $\bullet$ & $\bullet$ & $\bullet$ \\
\hline Hifas & $\bullet$ & - & $\bullet$ & $\bullet$ & $\bullet$ & - & $\bullet$ & $\bullet$ & $\bullet$ & - & - \\
\hline Traqueidas & - & - & $\bullet$ & $\bullet$ & $\bullet$ & - & $\mathbf{\square}$ & $\bullet$ & - & $\bullet$ & $\bullet$ \\
\hline Otros & & $\bullet$ & $\bullet$ & $\bullet$ & $\bullet$ & $\bullet$ & घ & $\bullet$ & $\bullet$ & $\bullet$ & $\bullet$ \\
\hline \multicolumn{12}{|l|}{ No-bioestructurados } \\
\hline Degradados & - & $\bullet$ & $\bullet$ & $\bullet$ & $\mathbf{\square}$ & $\mathbf{\square}$ & $\bullet$ & $\bullet$ & & & - \\
\hline Semiopacos & $\boldsymbol{\Delta}$ & $\boldsymbol{\Delta}$ & $\boldsymbol{\Delta}$ & $\boldsymbol{\Delta}$ & $\boldsymbol{\Delta}$ & $\boldsymbol{\Delta}$ & $\boldsymbol{\Delta}$ & $\boldsymbol{\square}$ & $\boldsymbol{\Delta}$ & $\boldsymbol{\Delta}$ & $\boldsymbol{\Delta}$ \\
\hline Fitoclastos opacos & 50,26 & 66,15 & 55,97 & 59,11 & 71,65 & 78,16 & 53,02 & 14 & 48,83 & 41,23 & 66,12 \\
\hline \multicolumn{12}{|l|}{ Equidimensionales } \\
\hline Redondeados & $\bullet$ & $\bullet$ & $\bullet$ & $\bullet$ & $\bullet$ & $\bullet$ & - & $\mathbf{\square}$ & $\bullet$ & $\bullet$ & $\bullet$ \\
\hline Subredondeados-angulares & घ & $\mathbf{\square}$ & घ & $\mathbf{\square}$ & घ & $\mathbf{\square}$ & - & घ & $\mathbf{\square}$ & $\mathbf{\square}$ & $\mathbf{\square}$ \\
\hline Angulares & $\boldsymbol{\Delta}$ & $\boldsymbol{\Delta}$ & $\Delta$ & $\boldsymbol{\Delta}$ & a & $\boldsymbol{\Delta}$ & $\boldsymbol{\Delta}$ & घ & $\mathbf{\square}$ & $\boldsymbol{\Delta}$ & $\boldsymbol{\Delta}$ \\
\hline \multicolumn{12}{|l|}{ Tablillas } \\
\hline Bioestructuradas & $\bullet$ & $\bullet$ & घ & $\bullet$ & $\bullet$ & $\bullet$ & $\bullet$ & - & $\bullet$ & $\bullet$ & $\bullet$ \\
\hline No-bioestructuradas & - & - & $\bullet$ & $\bullet$ & $\bullet$ & - & & - & $\bullet$ & $\mathbf{\square}$ & $\mathbf{\square}$ \\
\hline Charcoal & $\mathbf{\square}$ & $\bullet$ & $\bullet$ & $\mathbf{\square}$ & $\mathbf{a}$ & - & - & - & $\bullet$ & - & $\bullet$ \\
\hline MATERIA ORGÁNICA AMORFA & 18,8 & 9,4 & 5,4 & 4,8 & 6,2 & 1,67 & 31,4 & 16 & 18,4 & 14,8 & 9,4 \\
\hline Granular & $\boldsymbol{\Delta}$ & $\mathbf{\square}$ & - & $\bullet$ & $\mathbf{\square}$ & 口 & 口 & $\mathbf{\square}$ & 口 & $\boldsymbol{\Delta}$ & $\mathbf{\square}$ \\
\hline Granular-esponjosa & $\bullet$ & $\bullet$ & $\bullet$ & $\bullet$ & $\bullet$ & - & $\bullet$ & $\bullet$ & $\bullet$ & $\mathbf{\square}$ & $\mathbf{\square}$ \\
\hline Esponjosa & $\bullet$ & - & - & $\mathbf{\square}$ & $\bullet$ & $\mathbf{\square}$ & $\bullet$ & $\mathbf{\square}$ & $\bullet$ & - & $\mathbf{\square}$ \\
\hline Membranosa & $\bullet$ & $\bullet$ & - & $\mathbf{\square}$ & $\mathbf{\square}$ & $\mathbf{\square}$ & $\mathbf{\square}$ & - & $\bullet$ & $\mathbf{\square}$ & $\mathbf{\square}$ \\
\hline Fibrosa & - & - & - & - & - & - & $\mathbf{\square}$ & $\bullet$ & $\bullet$ & $\bullet$ & - \\
\hline Otros & - & $\Delta$ & $\Delta$ & $\mathbf{\square}$ & $\mathbf{\square}$ & - & $\bullet$ & - & $\Delta$ & $\bullet$ & - \\
\hline PALINOMORFOS & 3,4 & 0,6 & 2 & 0,6 & 1,2 & 0,67 & 22 & 54 & 4,8 & 0,8 & 5,6 \\
\hline Esporas y polen & 口 & $\boldsymbol{\Delta}$ & 口 & $\Delta$ & $\boldsymbol{\Delta}$ & $\boldsymbol{\Delta}$ & $\boldsymbol{\Delta}$ & $\bullet$ & $\boldsymbol{\Delta}$ & $\boldsymbol{\Delta}$ & $\boldsymbol{\Delta}$ \\
\hline Fitoplancton & $\bullet$ & - & - & - & - & - & $\bullet$ & $\boldsymbol{\Delta}$ & घ & - & $\bullet$ \\
\hline Indeterminados & घ & - & घ & - & - & - & $\bullet$ & $\bullet$ & - & - & $\mathbf{\square}$ \\
\hline Zooclastos & 0,2 & 0,2 & 0,4 & 0,2 & 0,2 & - & 0,2 & - & 0,2 & - & - \\
\hline
\end{tabular}



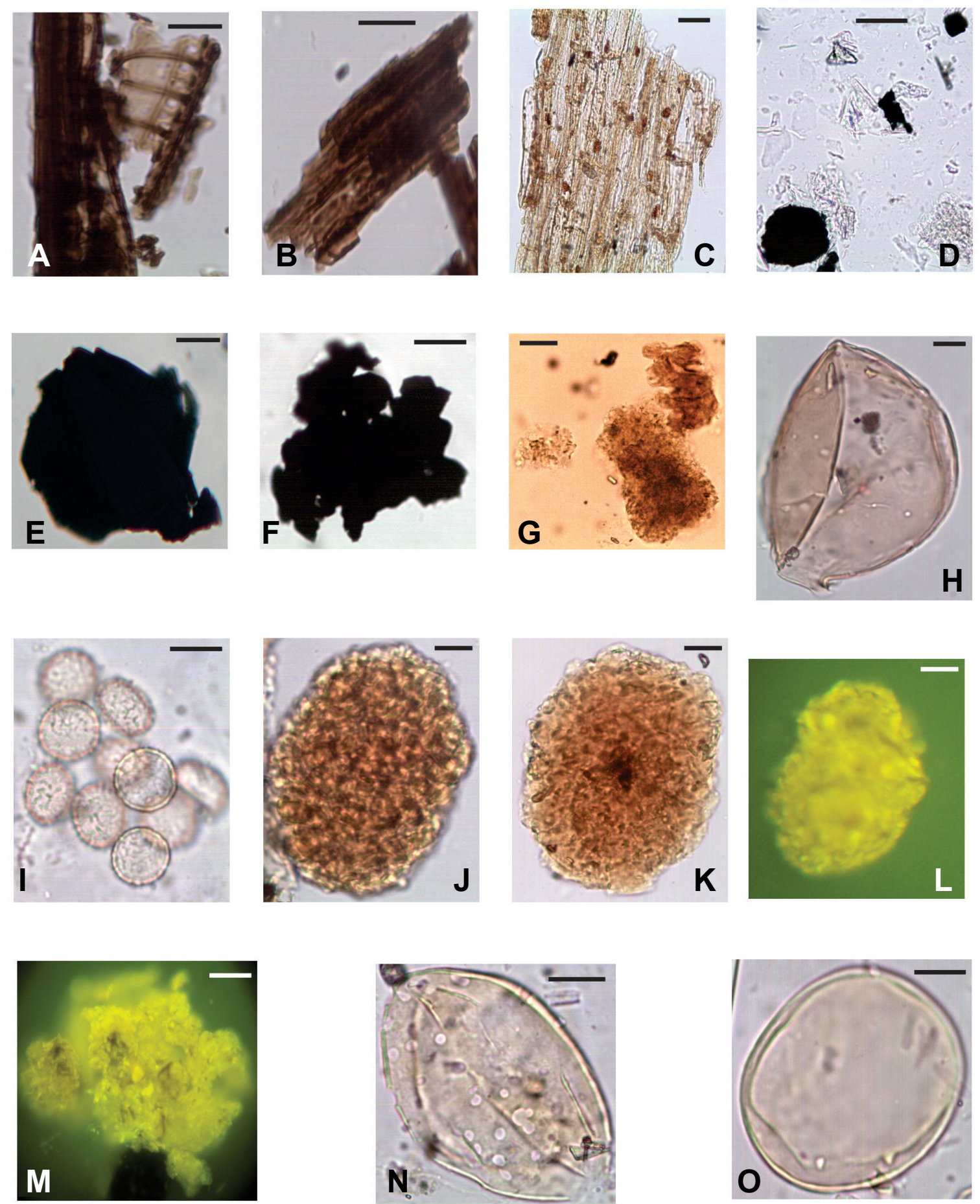

Figura 4. A-C, fitoclastos translúcidos bioestructurados; A-B, CTES-PMP $\mathrm{N}^{\circ}$ : 3074; C, CTES-PMP $\mathrm{N}^{\circ}$ : 3075. D-F, fitoclastos opacos; D, CTES-PMP N: 3084; E, CTES-PMP N: 3075; F, CTES-PMP N: 3085. G, Materia orgánica amorfa, CTES-PMP No: 3074. H, Glomus sp., CTES-PMP N ${ }^{\circ} 3074 \mathrm{e}: 38,1 / 95,5$. I, Lycoperdon sp., CTES-PMP N ${ }^{\circ} 3074 \mathrm{c}$ : 40,8/91,6. J-M, Colonias amorfizadas (sin estructura aparente) Botryococcus braunii Kützing, 1849; J-K, Microscopio óptico de luz transmitida, CTES-PMP N³075a: 39,6/106,2; 41,6/106,2; L-M, Microscopio de fluorescência, CTES-PMP N 3075b: 35,2/92,2; 42,3/93,2. N, Ovoidites parvus (Cookson \& Dettman) Nakoman, 1966, con evidencia de daño mecánico por corrosión o por actividad fúngica o bacteriana, CTES-PMP N ${ }^{\circ}$ 3074c: 41/83,7. O, Ovoidites sprigii (Cookson \& Dettmann) Zippi, 1998 CTES-PMP N 3074c: 44,7/88,2. Escalas: A, B, D, G, I-M = $10 \mu \mathrm{m} ; \mathrm{C}, \mathrm{H}, \mathrm{N}, \mathrm{O}=20 \mu \mathrm{m} ; \mathrm{E}, \mathrm{F}=5 \mu \mathrm{m}$.

Figure 4. A-C, biostructurated translucent phytoclasts; A-B, CTES-PMP $N^{\circ}: 3074$; C, CTES-PMP N: 3075 . D-F, opaque phytoclasts; D, CTESPMP N: 3084; E, CTES-PMP N: 3075; F, CTES-PMP No: 3085. G, amorphous organic matter, CTES-PMP N: 3074. H, Glomus sp., CTESPMP N 3074e: 38.1/95.5. I, Lycoperdon sp., CTES-PMP N 3074c: 40.8/91.6. J-M, amortized colonies (no apparent structure) Botryococcus braunii Kützing, 1849; J-K, transmitted light optical microscope, CTES-PMP N 3075a: 39.6/106.2; 41.6/106.2; L-M, fluorescence microscope, CTES-PMP N 3075b: 35.2/92.2; 42.3/93.2. N, Ovoidites parvus (Cookson \& Dettman) Nakoman, 1966, with evidence of mechanical damage by corrosion or fungal or bacterial activity, CTES-PMP N ${ }^{\circ}$ 3074c: 41/83.7. O, Ovoidites sprigii (Cookson \& Dettmann) Zippi, 1998 CTES-PMP $\mathrm{N}^{\circ}$ 3074c: 44.7/88.2. Scale bars: A-B, D, G, I-M $=10 \mu \mathrm{m} ; \mathrm{C}, \mathrm{H}, \mathrm{N}, \mathrm{O}=20 \mu \mathrm{m} ; \mathrm{E}-\mathrm{F}=5 \mu \mathrm{m}$. 


\section{DISCUSIÓN}

\section{Interpretación paleoambiental}

Palinofacies tipo I. Los fitoclastos traslúcidos no bioestructurados semiopacos y los fitoclastos opacos (Tabla 3 ), probablemente deriven de leños próximos a la fuente de origen, los que habrían sido oxidados, ya sea por exposición subaérea durante el transporte o bien dentro del mismo ambiente de depósito (Sebag et al., 2006), e incluso algunos habrían sufrido maduración térmica a causa de incendios (charcoal, sensu Scott, 2010). El predominio de fitoclastos opacos equidimensionales (angulares, subredondeadosangulares) denota escaso transporte y alta energía del medio. La materia orgánica amorfa es reducida, sugiriendo un ambiente relativamente oxidante. La ausencia de granos de polen de Tracheophyta puede deberse a distintos factores como $\mathrm{pH}$ alcalino, potencial redox (Eh) relativamente oxidante (al menos temporalmente) y/o a la intensa actividad biológica (Martínez et al., 2008). "Las condiciones alcalinas en zonas áridas provocan la destrucción de los palinomorfos hasta profundidades de varias decenas a centenares de metros..." (Volkheimer, 1972, ver Quattrocchio et al., 2003 , p.126). La proporción importante de materia orgánica oscurecida sugiere ambientes oxigenados alternativamente secos y húmedos, pero no saturados (Batten, 1996).

Palinofacies tipo II. El ambiente de depósito habría sido temporariamente anóxico y de poca profundidad, relativamente más reductor que el de la Palinofacies tipo I, dada la presencia de fitoclastos traslúcidos bioestructurados y materia orgánica amorfa bien preservada (Martínez et al., 2008). No obstante esto, los fitoclastos traslúcidos no bioestructurados semiopacos y los fitoclastos opacos presentes, habrían sufrido oxidación y escaso transporte (fitoclastos opacos equidimensionales angulares y subredondeadosangulares). Teniendo en cuenta los palinomorfos asociados en la M 8, probablemente las partículas amorfas esponjosas y membranosas podrían derivar de colonias degradadas de Botryococcus braunii. Cabe mencionar que en algunos ejemplares de Glomus sp., Zygnemataceae y Equisetosporites sp., así como también en partículas traslúcidas y opacas, se observan perforaciones de diferentes tamaños relacionadas probablemente a biodegradación producto de la actividad fúngica y/o bacteriana. Las esporas de hongos indican cercanía a la fuente de origen. Por la alta frecuencia relativa de Botryococcus braunii en la M 8, se habrían desarrollado en un cuerpo ácueo bien oxigenado, con una carga de nutrientes entre oligo- a mesotrófica y quizás eurihalino (Martínez et al., 2008).

\section{Palinomorfos}

Los palinomorfos habrían integrado dos paleocomunidades: acuática, conformada por Botryococcus braunii, Ovoidites sprigii y Ovoidites parvus, y terrestre, representada por Glomus sp., Lycoperdon sp., Equisetosporites sp. y Chenopodipollis chenopodiaceioides.

La paleocomunidad acuática se habría desarrollado en cuerpos de agua dulceacuícolas poco profundos. Las colonias de Botryococcus braunii (M 7 y 8) son compuestas simples (no ramificadas), de forma ovoide, tamaño uniforme y en general con buena preservación. Sin embargo otras colonias (o partes de las mismas) se observan amorfizadas o aparentemente degradadas. Dada la alta frecuencia relativa y las características morfológicas de las mismas (compuestas simples no ramificadas) se infiere que ellas corresponderían a los primeros estadíos de desarrollo (juveniles) (GuyOhlson, 1992; Rodríguez Amenábar \& Ottone, 2003). Las condiciones paleoecológicas favorables para su presencia habrían obedecido a que el cuerpo ácueo estuvo oxigenado, con salinidad $\leq 35 \%$ y pH bajo (Tyson, 1995). Estas condiciones habrían tenido una duración efímera y podrían haberse tornado poco favorables o adversas (quizás, por anoxia), provocando el detenimiento del crecimiento de las colonias y su posterior degradación (por la presencia de colonias amorfizadas) (Guy-Ohlson, 1992; Rodríguez Amenábar \& Ottone, 2003). En la actualidad el género se distribuye en regiones cálidas, tropicales y templadas. En cuanto a las Zygnemataceae, las especies actuales originan sus esporas en aguas mesotróficas, ricas en oxígeno y relativamente cálidas (Van Geel, 1978, 2001; Van Geel \& van der Hammen, 1978), no obstante esto las esporas de resistencia son capaces de preservarse bajo situaciones de stress hídrico (Borel et al., 2003). Las colonias de Botryococcus braunii y las esporas de Zygnemataceae se encuentran asociados solamente en la muestra M 7.

La paleocomunidad terrestre, habría estado integrada en parte por elementos del suelo como Glomus sp. y Lycoperdon sp. (teniendo en cuenta los hábitos de los taxones actuales afines), y vegetación xerófila, Equisetosporites sp. y Chenopodipollis chenopodiaceioides. En el caso de Glomus sp., habría formado micorrizas arbusculares o endomicorrizas con plantas superiores (Chenopodiceae, Fagaceae, Poaceae, entre otras) (Honrubia, 2009). En la actualidad el micelio es muy eficiente en la movilización de fósforo insoluble del suelo y en el traslado del mismo a la planta. Dado que el escaso o nulo aporte de fósforo es a menudo un factor limitante para el crecimiento de las plantas, estos hongos son vitales en muchos hábitats naturales ayudando a las plantas a prosperar en suelos pobres (Kendrick, 2000; Becerra et al., 2007; Martínez \& Pugnaire, 2009). Por lo tanto su presencia indica suelos asociados a condiciones de aridez o estrés ambiental (Limaye et al., 2007). Los hallazgos fósiles (esporas, restos de arbúsculos, etc) más antiguos asignados al género Glomus datan del Paleozoico Temprano (Honrubia, 2009; Taylor et al., 2009). A pesar de estos registros tan antiguos, la presencia del género es más frecuente en sedimentos Cenozoicos. En Argentina, se cita para el Oligoceno/Mioceno (Náñez et al., 2009) y Pleistoceno-Holoceno (Garralla, 2003; Grill \& Morrás 2010; Musotto et al., 2012, 2013; entre otros). Lycoperdon sp. se habría desarrollado probablemente sobre madera en descomposición (como saprófito), aunque en la actualidad algunas especies también forman ectomicorrizas (Kendrick, 2000). Los Lycoperdales actuales, producen una masa de esporas que son expulsadas al exterior del peridio a 
través de un poro (ostiolo) apical pudiendo ser diseminadas por el viento (Kendrick, 2000). Las altas frecuencias relativas de las esporas en especial de Lycoperdon sp., se deberían a que la esporulación se produjo próxima a la fuente de depósito de las mismas (Tyson, 1995). El primer registro de esporas asignadas al género Lycoperdon o al menos al orden Lycoperdales data del Cretácico TardíoEoceno de Gran Bretaña (Jarzen \& Elsik, 1986). Es citado también para el Neógeno de Norte América (Elsik, 1976) y para el Holoceno de África (Jarzen \& Elsik, 1986) y Brasil (Garcia, 1994). Los ejemplares estudiados representan hasta el momento, el primer registro del género en sedimentos del Mioceno de Argentina.

A pesar de la escasa representación de Equisetosporites sp. (Ephedraceae) y Chenopodipollis chenopodiaceioides (Chenopodiaceae) (menos de 10 ejemplares en la M 7) se considera la presencia de ambas especies, por ser integrantes recurrentes de las paleopalinofloras del Mioceno de Argentina. En la actualidad la familia Ephedraceae que es monotípica, posee una amplia distribución geográfica en regiones áridas en latitudes templado cálidas, y también en zonas desérticas o montañosas, desarrollando subarbustos, arbustos hasta pequeños árboles erectos trepadores o rastreros rizomatosos (Hunziker \& Novara, 1998; Zuloaga et al., 2008). La familia Chenopodiaceae que es cosmopolita (exceptuando los polos), presenta arbustos, hierbas anuales y perennes (raro, pequeños árboles o plantas rizomatosas o rastreras radicantes), particularmente abundantes en sitios xerófitos o salobres (Tolaba, 2006; Zuloaga et al., 2008). Por lo expuesto podríamos inferir que la vegetación circundante habría tenido entre sus integrantes al menos formas herbáceas y arbustivas, desarrolladas en un ambiente semiárido o xérico bajo un clima cálido. No obstante esto, Anzótegui et al. (2007) infieren para otra localidad (Río Totoral) de la Aloformación Playa del Zorro, en base al registro de vegetación palustre e higrófila, condiciones ambientales locales más húmedas desarrolladas bajo un clima cálido con estacionalidad seca. De la misma manera al comparar el registro palinológico de las formaciones Chiquimil (Mautino, 2007, 2010, 2011; Mautino \& Anzótegui, 1998, 2000, 2002a,b, 2014) y Palo Pintado (Anzótegui \& Cuadrado, 1996; Acevedo et al., 1997, 1998; Mautino, 2007; Horn, 2014; Horn et al., 2011a,b) (Mioceno tardío/Plioceno), con el de la localidad en estudio, se observa que presentan un alto número de ejemplares y una gran diversidad de especies (en especial la Formación Chiquimil) que habrían conformado las paleocomunidades hidrófita (planctónica y palustre), higrófita, halófita xerófita, y boscosa de altura, también desarrolladas bajo condiciones ambientales semejantes a las de Río Totoral. Por lo tanto, las condiciones paleoambientales notoriamente más áridas de La Cascadita, podrían obedecer a la acción de un conjunto de determinados factores que se describen en el siguiente apartado.

\section{Paleoambiente de sedimentación}

En relación a la litología y granulometría de la columna sedimentaria estudiada (Figura 2), y de acuerdo con Parra \& Morales (2003), se infiere que la alternancia de sedimentos finos y arenosos podría sugerir variaciones en la energía, el nivel de agua, y el avance o retroceso de los bordes del cuerpo ácueo ocasionados, por cambios climáticos estacionales o por corrientes hiperconcentradas provocadas por la inestabilidad tectónica de la región andina durante el Mioceno Tardío. Puntualmente el continuo ascenso de la Sierra de Quilmes o del Cajón durante la sedimentación de estos depósitos, modificó la dirección del sistema de drenaje (de E a SE) (Bossi et al., 2000) y actuó también como barrera para los vientos (Georgieff et al., 2012) generando condiciones climáticas cada vez más áridas (Bossi et al., 2000). A su vez la presencia de ceniza volcánica, sugiere un aporte desde un centro volcánico cercano, demostrando que la actividad volcánica fue coetánea a la sedimentación. La ceniza depositada en el área, probablemente modificó durante su circulación, de manera temporal, la composición de la atmósfera (Fúster Casas, 2000) y durante su depósito, al paleoambiente.

\section{CONCLUSIONES}

A partir del análisis palinofacial, en La Cascadita (Aloformación Playa del Zorro) se distinguen dos tipos de Palinofacies. La Palinofacies Tipo I, se caracteriza por la alta frecuencia relativa de fitoclastos traslúcidos bioestructurados semiopacos y fitoclastos opacos equidimensionales que indican ambientes oxidantes y denotan escaso transporte y alta energía del medio. La ausencia de granos de polen puede deberse a distintos factores como $\mathrm{pH}$ alcalino, potencial redox (Eh) relativamente oxidante (quizá temporalmente) y a la intensa actividad biológica. La materia orgánica amorfa es reducida. La Palinofacies Tipo II, se caracteriza por las altas frecuencias relativas de palinomorfos y materia orgánica amorfa; a su vez presenta los más bajos valores de fitoclastos. Los fitoclastos traslúcidos bioestructurados están mejor representados por cutículas con alta frecuencia relativa. El ambiente de depósito habría sido temporariamente anóxico y de poca profundidad, dada la presencia de fitoclastos bioestructurados y materia orgánica amorfa bien preservada. La alta frecuencia relativa de Botryococcus braunii (M 8) sugiere la existencia de un cuerpo ácueo oxigenado, de bajo $\mathrm{Ph}$ y con una carga de nutrientes entre oligo- a mesotrófica, quizás eurihalino. El paleoclima regional habría sido cálido, con estacionalidad seca, siendo esta última muy marcada en la localidad La Cascadita.

\section{AGRADECIMIENTOS}

Agradecemos muy especialmente a R. Herbst por su colaboración en el campo para la extracción de las muestras palinológicas y a los revisores del manuscrito por sus valiosos aportes. El presente trabajo se articula con los proyectos de investigación PICTO-2011/216 P. BID 2013-2016 (Agencia Nacional de Promoción Científica y Tecnología/ Universidad Nacional del Nordeste) y PI F021 Res. 921/10 (Universidad Nacional del Nordeste). 


\section{REFERENCIAS}

Acevedo, L.T.; Anzótegui, L.M. \& Mautino, L.R. 1998. Análisis palinológico preliminar de la Formación Palo Pintado (Mioceno superior), en Quebrada del Estanque, Salta, Argentina. In: COMUNICACIONES CIENTÍFICAS Y TÉCNOLÓGICAS, 1998. Resúmenes expandidos, Corrientes, Universidad Nacional del Nordeste, v. 3, p.143-146.

Acevedo, T.L.; Mautino, L.R.; Anzótegui, L.M. \& Cuadrado, G.A. 1997. Estudio palinológico de la Formación Palo Pintado (Mioceno superior), Provincia de Salta, Argentina. Parte II esporas. Geociencias, 2:112-120.

Anzótegui, L.M. \& Cuadrado, G.A. 1996. Palinología de la Formación Palo Pintado, Mioceno Superior, Provincia de Salta, República Argentina. Parte I, taxones nuevos. Revista Española de Micropaleontología, 28:77-92.

Anzótegui, L.M.; Garralla, S.S. \& Herbst, R. 2007. Fabaceae de la Formación El Morterito, (Mioceno Superior) del valle del Cajón, provincia de Catamarca, Argentina. Ameghiniana, 44:183-196.

Batten, D.J. 1996. Palynofacies and palaeoenviromental interpretations. In: J. Jansonius \& D.C. McGregor (eds.) Palynology: Principles and Applications, American Association of Stratigraphic Palynologists Foundation, v. 3, p. 1011-1064.

Becerra, A.G.; Arrigo, N.M.; Bartolini, N.; Domínguez, L.S. \& Cofré, M.N. 2007. Arbuscular mycorrhizal colonization of Alnus acuminata Kunth in northwestern Argentina in relation to season and soil parameters. Ciencia Suelo, 25:7-13.

Borel, C.M.; Guerstein, G.R. \& Prieto, A.R. 2003. Palinomorfos acuáticos (algas y acritarcos) del Holoceno de la laguna Hinojales (Buenos Aires, Argentina): interpretación paleoecológica. Ameghiniana, 40:531-544.

Bossi, G.E.; Muruaga, C.M.; Sanagua, J.G.; Hernando, A.B.; Quiroga, G.; Ahumada, A.L. \& Graviloff, I. 1992. Aloestratigrafía del Neógeno del valle del Cajón (Provincia de Catamarca). In: REUNIÓN ARGENTINA DE SEDIMENTOLOGÍA, 4, 1992. Actas, La Plata, p. 137-144.

Bossi, G.E.; Vides, M.E.; Georgieff, S.M.; Muruaga, C.M. \& Ibáñez, L.M. 2000. Análisis delas paleocorrientes y de la varianza de los componentes a tres niveles, Neógeno del valle del Cajón, Catamarca, Argentina. Revista de la Asociación Argentina de Sedimentología, 7:23-47.

Elsik, W.C. 1976. Microscopic fungal remains and Cenozoic palynoestratigraphy. Geoscience and Man, 15:115-120.

Esteban, G.I. 1999. Nuevo registro de Sphenotherus (Xenarthra, Mylodontidae) en el Terciario superior del valle de El Cajón (provincia de Catamarca, Argentina): revisión sistemática del género. Ameghiniana, 36:317-321.

Esteban, G.I. \& Nasif, N.L. 1996. Nuevos Dasypodidae (Mammalia, Xenarthra) del Mioceno tardío del Valle del Cajón, Catamarca, Argentina. Ameghiniana, 33:327-334.

Fúster Casas, J.M. 2000. Vulcanismo y cambio climático. In: Horizontes culturales, las fronteras de la ciencia, Barcelona, Editorial Espasa Calpe, p. 115-128 (Programa de Promoción de la Cultura Científica y Tecnológica 1998).

Garcia, M.J. 1994. Palinologia de turfeiras quaternárias do médio vale do rio Paraíba do Sul, Estado de São Paulo, São Paulo, Brasil. Programa de Pós-Graduação em Geologia Sedimentar, Universidade de São Paulo, Tese de Doutorado, 354 p.

Garralla, S.S. 2003. Estudio palinológico en secuencias sedimentarias turbosas del Holoceno de los Valles Calchaquies, Argentina. Programa de Posgrado en Biología, Universidad Nacional del Nordeste, Tesis Doctoral, 219 p.
Georgieff, S.M.; Sosa Gómez, J. \& Schiuma, M. 2012. Análisis estratigráfico-estructural del neógeno de Catamarca, Tucumán, Salta y Santiago del Estero. Ameghiniana, 49:10-11.

Grill, S.C. \& Morrás, H.J.M. 2010. Análisis palinofacial de sedimentos del Cenozoico tardío en la Pampa Ondulada (Argentina): primeros resultados. Revista Brasileira de Paleontología, 13:221-232. doi:10.4072/rbp.2010.3.06

Guy-Ohlson, D. 1992. Botryococcus as an aid in the interpretation of paleoenviroment and depositional processes. Review of Palaeobotany and Palynology, 71:1-15. doi:10.1016/00346667(92)90155-A

Honrubia, M. 2009. Las micorrizas: una relación planta-hongo que dura más de 400 millones de años. Anales del Jardín Botánico de Madrid, 66:133-144. doi:10.3989/ajbm.2226

Horn, M.Y. 2014. Palinofloras de las formaciones El Morterito y Palo Pintado (Mioceno Superior-Plioceno Inferior), noroeste de Argentina. Programa de Posgrado en Ciencias Naturales, Universidad Nacional de La Plata, Tesis Doctoral, 141 p.

Horn, M.Y.; Galli, C.I.; Mautino, L.R. \& Anzótegui, L.M. 2011 a. Palinología y litofacies de la Formación Palo Pintado (Mioceno Superior), en las localidades Río Calchaquí y Quebrada El Estanque, Salta Argentina. Ameghiniana, 48:15-16.

Horn, M.Y.; Mautino L.R.; Galli, C.I. \& Anzótegui, L.M. 2011 b. Palinomorfos de la Formación Palo Pintado (Mioceno Superior), en la localidad Río Calchaquí, Salta Argentina. In: COMUNICACIONES CIENTÍFICAS Y TECNOLÓGICAS, 2011. Resúmenes, Resistencia, Universidad Nacional del Nordeste, Disponible en http://www.unne.edu.ar/unnevieja/ investigacion/com2011/CB-Web/CB-027.pdf; accesado en 17/04/2016.

Hunziker, J.H. \& Novara, L.J. 1998. Ephedraceae Dumort. Aportes Botánicos de Salta, 5:1-14.

Jarzen, D.M. \& Elsik, W.C. 1986. Fungal palynomorphs recovered from recent river deposits, Luangwa Valley, Zambia: Palynology, 10:35-60. doi:10.1080/01916122.1986.9989302

Kendrick, B. 2000. The Fifth Kingdom. $3^{\text {a }}$ ed. Newburyport, Focus Publishing, 371p.

Kützing, F.T. 1849. Species algarum. Leipzig, Brockhaus, 922 p.

Limaye, R.B.; Kumaran, K.P.N.; Nair, K.M. \& Padmaiai, D. 2007. Non-pollen palynomorphs as potential palaeoenvironmental indicators in the Late Quaternary sediments of the west coast of India. Current Science, 92:1370-1382.

Martínez, M.A.; Ferrer, N. \& Asensio, M. 2008. Primer registro de algas dulceacuícolas del Paleógeno de la Cuenca de Nirihuau, Argentina: descripciones sistemáticas y análisis palinofacial. Ameghiniana, 45:719-735.

Martínez, L.B. \& Pugnaire, F.I. 2009. Interacciones entre las comunidades de hongos formadores de micorrizas arbusculares y de las plantas. Algunos ejemplos en los ecosistemas semiáridos. Ecosistemas, 18:44-54.

Mautino, L.R. 2007. Chlorophyta de los Valles Calchaquíes (Mioceno Medio y Superior), Argentina. Revista Española de Micropaleontología, 39:81-102.

Mautino, L.R. 2010. Palinofloras de las Formaciones San José y Chiquimil (Mioceno Medio y Superior), Noroeste de Argentina. Programa de Posgrado en Biología, Universidad Nacional del Nordeste, Tesis Doctoral, $444 \mathrm{p}$.

Mautino, L.R. 2011. Nuevas especies de las formaciones San José y Chiquimil (Mioceno Medio y Superior), Noroeste de la Argentina. Revista Brasileira de Paleontologia, 14:279-290. doi:10.4072/rbp.2011.3.07 
Mautino, L.R. \& Anzótegui, L.M. 1998. Palinología de la Formación Chiquimil (Mioceno superior) Localidad Vallecito, Provincia de Catamarca. Parte 1: Especies Nuevas. Ameghiniana, 35:227-233.

Mautino, L.R. \& Anzótegui, L.M. 2000. Esporas del Mioceno y retrabajadas del Mesozoico en la Formación Chiquimil, Vallecito, Provincia de Catamarca. Ameghiniana, 37:13-22.

Mautino, L.R. \& Anzótegui, L.M. 2002a. Palinología de la Formación Chiquimil (Mioceno Superior) Vallecito, provincia de Catamarca. Parte 2. Polen. Ameghiniana, 39:257-270.

Mautino, L.R. \& Anzótegui, L.M. 2002b. Palinología de la Formación Chiquimil Mioceno Superior), Vallecito, provincia de Catamarca. Parte 3. Polen. Ameghiniana, 39:271-284.

Mautino, L.R. \& Anzótegui, L.M. 2014. Novedades palinológicas de las formaciones San José y Chiquimil (Mioceno medio y tardío), noroeste de Argentina. Revista del Museo Argentino de Ciencias Naturales, 16:143-164.

Morton, L.S. 2004. Taxonomía y paleoecología de la malacofauna (Bivalvia-Gastropoda) dulceacuícola del Neógeno, en los valles de Santa María y del Cajón, Catamarca y Tucumán, Argentina. Programa de Posgrado en Biología, Universidad Nacional del Nordeste, Tesis Doctoral, 134 p.

Morton, L.S. \& Herbst, R. 2012. Moluscos fósiles continentales (Bivalvia-Gastropoda) del NOA Y NEA de Argentina. Ameghiniana, 49:14.

Musotto, L.L.; Bianchinotti, M.V. \& Borromei, A.M. 2012. Pollen and fungal remains as environmental indicators in surface sediments of Isla Grande de Tierra Del Fuego, sourthernmost Patagonia. Palynology, 36:162-179. doi:10.1080/01916122.2 012.662919

Musotto, L.L; Bianchinotti, M.V. \& Borromei, A.M. 2013. Inferencias paleoecológicas a partir del análisis de microfósiles fúngicos en una turbera pleistoceno-holocena de Tierra del Fuego, Argentina. Revista del Museo Argentino de Ciencias Naturales, 15:89-98.

Nakoman, E. 1966. Contribution a l'étude palynologigue des formations tertiaires du Bassin de Thrace. Annales de la Societé Géologique du Nord, 46:65-107.

Náñez, C.; Quattrocchio, M.E. \& Ruiz, L. 2009. Palinología y micropaleontología delas Formaciones San Julián y Monte León (Oligoceno-Mioceno temprano) en el subsuelo de cabo Curioso, provincia de Santa Cruz, Argentina. Ameghiniana, 46:669-693.

Parra, L.E. \& Morales, J.A. 2003. Descripción e interpretación de la columna estratigráfica tipo de la serie vulcano-sedimentaria neógena del Valle del Cajón (Catamarca, Argentina). Geogaceta, 33:55-58.

Quattrocchio, M.; Durango de Cabrera, J. \& Galli, C. 2003. Formación Anta (Mioceno Temprano), Subgrupo Metán (Grupo Orán), en el río Piedras, Provincia de Salta. Datos palinológicos. Revista de la Asociación Geológica Argentina, 58:117-127.

Rodríguez Amenábar, C. \& Ottone, E.G. 2003. La aplicación de Botryococcus (Chlorococcales) como indicador paleoambiental en el Triásico de Argentina. Revista Española de Micropaleontología, 35:161-169.

Scott, A.C. 2010. Charcoal recognition, taphonomy and uses in palaeoenvironmental analysis. Palaeogeography,
Palaeoclimatology, Palaeoecology, 291:11-39. doi:10.1016/j. palaeo.2009.12.012

Sebag, D.; Copard, Y.; Di-Giovanni, Ch.; Durand, A.; Laignel, B.; Ogier, S. \& Lallier-Verges, E. 2006. Palynofacies as useful tool to study origins and transfers of particulate organic matter in recent terrestrial environments: synopsis and prospects. Earths Science Reviews, 79:241-259. doi:10.1016/j.earscirev.2006.07.005

Taylor, T.N.; Taylor, E.L. \& Krings, M. 2009. Paleobotany. The Biology and Evolution of fossil plants. $2^{\mathrm{a}}$ ed. New York, Elsevier, $1253 \mathrm{p}$.

Tolaba, J.A. 2006. Chenopodiaceae Vent. Aportes Botánicos de Salta, 7:1-49.

Truswell, E.M.; Sluiter, I.R. \& Harris, W.K. 1985. Palynology of the Oligocene-Miocene sequence in the Oakvale-1 corehole, western Murray Basin, South Australia. Bureau of Mineral Resources, Journal of Australian Geology and Geophysics, 9:267-295.

Turner, J.C. 1973. Descripción Geológica de la Hoja 11d, Laguna Blanca, Provincia de Catamarca, escala 1:200.000. Carta Geológico-Económica de la República Argentina. Buenos Aires, Servicio Nacional Minero Geológico, 71 p. (Boletín 142).

Tyson, R.V. 1995. Sedimentary organic matter. Organic facies and palynofacies. Oxford, Chapman and Hall, 615 p.

Van Geel, B. 1978. A paleoecologycal study of Holocene peat bog sections in Germany and the Netherlands, based on one analysis of pollen and macro-and microscopic remains of fungi, algae, cormophytes and animals. Review of Palaeobotany and Palynology, 25:1-120. doi:10.1016/0034-6667(78)90040-4

Van Geel, B. 2001. Non-pollen palynomorphs. In: J.P. Smol; H.J.B. Birks \& W.M. Last (eds.) Tracking environmental change using lake sediments, Terrestrial, algae, and siliceus indicators, Kluwe Academic Publisher, p. 99-119.

Van Geel, B. \& van der Hammen, T. 1978. Zygnemataceae in Quaternary Colombian sediments. Review of Palaeobotany and Palynology, 25:377-392. doi:10.1016/0034-6667(78)90021-0

Vides, M.E. 2009. La sucesión Neógena aflorante entre Peñas Azules y Ovejería Chica en el valle del Cajón, Catamarca. Acta Geológica Lilloana, 21:57-65.

Vides, M.E. \& Bazán, C.A. 2004. La Aloformación Playa del Zorro, Quebrada del Totoral, valle del Cajón, provincia de Catamarca. In: REUNIÓN ARGENTINA DE SEDIMENTOLOGÍA, 10, 2004. Resúmenes expandidos, San Luis, p. 172-173.

Volkheimer, W. 1972. Palinomorfos como fósiles guía (Primera Parte). Técnicas de laboratorio palinológico. Revista Minera Geología y Mineralogía, 30:17-21.

Zippi, P.A. 1998. Freshwater algae from the Mattagami Formation (Albian), Ontario: Paleoecology, botanical affinities and systematic taxonomy. Micropaleontology, 44:1-78. doi: $10.2307 / 1485998$

Zuloaga, F.O.; Morrone, O. \& Belgrano, M. 2008. Catálogo de las plantas vasculares del Cono Sur, Dicotyledoneae: AcanthaceaeFabaceae (Abarema-Schizolobium). St. Louis, Missouri Botanical Garden, 1303 p. (Monographs in Systematic Botany 107).

Received in April, 2015; accepted in February, 2016. 